\title{
Hábitos Deformantes Orales en Preescolares y Escolares: Revisión Sistemática
}

\author{
Mouth Deforming Habits Present in Preschoolers and School Children: \\ Systematic Review
}

\author{
Sandra Susana Parra Iraola \& Andrea Gissela Zambrano Mendoza
}

PARRA, I. S. S. \& ZAMBRANO, M. A. G. Hábitos deformantes orales en preescolares y escolares: Revisión sistemática. Int. J. Odontostomat., 12(2):188-193, 2018.

RESUMEN: Los hábitos deformantes orales son una de las principales causas de las maloclusiones, entre los más frecuentes tenemos: la succión digital, el empuje lingual, la onicofagia, la queilofagia, la respiración oral y la masticación de objetos. El objetivo de este estudio fué efectuar una evaluación y análisis de la evidencia científica disponible actualizada en los últimos 7 años sobre los hábitos deformantes orales en preescolares y escolares de 3 a 12 años de edad. Se realizó una revisión sistemática de la literatura en bases de datos referativos como: Scielo, LILACS, GOOGLE ACADÉMICO, en artículos publicados sobre hábitos orales deformantes presentes en niños preescolares y escolares de 3 a 12 años de edad, con fechas de publicación que datan del 2010 al 2017, no hubo restricción en cuanto a sexo o zona geográfica. Se encontró una alta incidencia de hábitos deformantes orales en países de América latina, Centro américa y el Caribe; en países como Cuba la incidencia fue muy alta, el hábito que tuvo mayor prevalencia en la muestra fue la Onicofagia seguido de la disfunción lingual, resultados similares se reflejaron en países como Venezuela, México y Colombia. Los resultados de esta revisión evidencian la importancia del estudio de los hábitos deformantes orales, los cuales constituyen una de las principales causas de las maloclusiones, que a su vez son el tercer problema de salud bucodental. En su gran mayoría, de los artículos tomados como referencia demostraron que no existe una diferencia estadísticamente significativa respecto al sexo y la prevalencia de hábitos parafuncionales. Se destaca la importancia de implementar nuevos proyectos de investigación respecto a los hábitos parafuncionales en la población preescolar y escolar, acompañado de programas educativo en nuestro país Ecuador, ya que en la actualidad fueron pocas las publicaciones que pudimos tomar de referencia.

PALABRAS CLAVE: hábitos orales deformantes, hábitos orales, dentición temporaria, dentición mixta, maloclusión.

\section{INTRODUCCIÓN}

Numerosas son las causas que pueden originar alteraciones o acelerar las posiciones incorrectas de los dientes y sus relaciones inadecuadas con los maxilares, entre las que se encuentran elementos totalmente externos sobre los cuales se puede actuar; dentro de estas causas, se pueden mencionar los hábitos orales deformantes (Cepero Sánchez et al., 2007).

La literatura científica define un hábito como una costumbre o práctica que se adquiere mediante la repetición frecuente de un mismo acto que llega a generar satisfacción. En un principio un hábito puede ser consciente y luego convertirse en inconsciente (Chamorro et al., 2016).
Los hábitos pueden clasificarse en 2 grupos:

- Beneficiosos o funcionales: son aquellos cuya práctica de una función normal realizada correctamente, estimula y beneficia el desarrollo. La masticación, la deglución y la respiración normales son ejemplos de ellos.

- Perjudiciales o deformantes: son los que resultan de una función normal o que se adquieren por prácticas repetitivas de un acto que no es funcional ni necesario; por ejemplo: la succión digital, el empuje lingual, la onicofagia, la queilofagia, la respiración oral y la masticación de objetos (Cepero Sánchez et al.; Chamorro et al.). 
Estos hábitos son de indudable causa primaria o secundaria de maloclusiones o deformaciones dentomaxilofaciales (Cepero Sánchez et al.).

Las maloclusiones o deformaciones dentoesquelética ocupan el tercer lugar en las alteraciones odontológicas, después de la caries y la enfermedad periodontal; pueden alterar el desarrollo normal del sistema estomatognático, conllevando a una deformación ósea que va a tener menor o mayor repercusión según la edad en que se inicia el hábito (Cepero Sánchez et al.; Ruiz Feria et al., 2014; Aróstica \& Carrillo, 2016; Andrade Roman, 2016).

Dentro de la etiología de la maloclusión, los hábitos orales deletéreos de la musculatura orofacial son puntos clave, por lo que se considera necesaria la prevención, interrupción y tratamiento interceptivo precoz mediante su modificación (Ruiz Feria et al.).

Habito de succión digital: es uno de los hábitos orales deformantes más frecuentes en el niño, capaz de producir grandes anomalías dentomaxilofaciales, alteraciones en la función masticatoria, en la psiquis y del estado de salud general. La succión del pulgar es la forma más común, aunque se pueden encontrar casos de succión de otros dedos y en una variada gama de posiciones. ( Acosta et al., 2013).

Consiste en la introducción de uno o más dedos de una de las manos del niño dentro de la cavidad oral (generalmente el primer dedo), y que se realiza usualmente con la yema o pulpejo del dedo apoyado sobre la bóveda palatina, el proceso alveolar incisivo y la cara lingual o palatina de los incisivos superiores. La colocación del dedo entre las arcadas las mantiene separadas y la lengua se mantiene en contacto presionando durante el acto de succión, reforzando la acción de este (Cepero Sánchez et al.).

Empuje o Protracción lingual: es la acción de empujar o proyectar la lengua hacia delante, que se produce en el momento de realizar la deglución. Se clasifica en simple, compleja y complicada (persistencia del patrón de deglución infantil), de acuerdo con sus características clínicas y etiológicas, el segundo y tercer caso deberá ser tratada por el ortodoncista (Martín Zaldivar et al., 2010).

- Protracción lingual simple: se caracteriza por presentar una mordida abierta anterior muy bien definida. Hay buena adaptación oclusal de ambas arcadas.
- Protracción lingual compleja: presenta una mordida abierta anterior amplia y mal delimitada, no hay contacto entre las arcadas dentarias durante la deglución y la adaptación oclusal de ambas arcadas presenta gran inestabilidad a nivel de incisivo, canino y premolares.

- Protracción lingual complicada: también conocida como deglución infantil conservada o persistencia de la deglución infantil. Solo hay contacto oclusal entre ambas arcadas en el estadio final de la deglución, entre los últimos molares de cada cuadrante (Cepero Sánchez et al.).

Respiración oral: Se observa en los pacientes que tienen interferencias en la función respiratoria normal de las vías aéreas nasales, como consecuencia de enfermedad local general, ya sea obstrucción nasal por un proceso inflamatorio crónico, adenoiditis, asma u otros. Pueden ser de 3 tipos: temporaria, en el caso de un resfriado o catarro normal; estacional, asociado con alergias nasorespiratorias y crónicas, en pacientes con obstrucciones adenoideas, dando origen a un labio superior corto, con inadecuado cierre bilabial. La respiración oral constituye un síndrome, cuyo diagnóstico definitivo deberá ser realizado por el otorrinolaringólogo, el estomatólogo solo puede hacer un diagnóstico presuntivo (Cepero Sánchez et al.).

Succión o mordisqueo del labio (queilofagia): Es la costumbre como su nombre lo indica, de mordisquear o chuparse el labio, generalmente el inferior. Produce retracción de la mandíbula durante el acto, linguoversión de incisivos inferiores y vestibuloversión de incisivos superiores. La queilofagia está definida por el Diccionario Terminológico de Ciencias Médicas como: hábito morboso o tic de morderse los labios (Cepero Sánchez et al.).

Onicofagia: Se define como el hábito de comer o morder las uñas con los dientes, afectando los tejidos blandos que las rodean, tales como la cutícula y piel de los dedos. Es bastante común en niños y adultos jóvenes, sin embargo, se observa con baja frecuencia antes de los 4 años, puesto que la mayor parte de los casos ocurren entre los 4 a 6 años. Puede producir desviación de uno o más dientes, desgaste dentario localizado y afectación localizada del tejido periodontal (Cepero Sánchez et al.; Chamorro et al.).

La presente revisión sistemática tuvo como objetivo efectuar una evaluación y análisis de la evidencia científica disponible actualizada sobre los hábitos 
deformantes orales en niños preescolares y escolares de 3 a 12 años de edad. Se encontró poca información sobre este tema en nuestro país Ecuador, esta problemática nos motivó a escribir al respecto.

\section{MATERIAL Y MÉTODO}

Para satisfacer la resolución de esta problemática, se realizó una revisión sistemática de la literatura en bases de datos referativos como: Scielo, LILACS, GOOGLE ACADÉMICO en artículos publicados sobre hábitos orales deformantes presentes en niños de edad preescolares y escolares de 3 a 12 años de edad, se revisaron artículos con fechas de publicación que datan del 2010 al 2017, no hubo restricción en cuanto a sexo o zona geográfica.

La búsqueda se ejecutó simultáneamente por dos investigadores, en forma independiente, utilizando estrategias simples y específicas. Los encabezados temáticos y palabras claves utilizadas fueron: hábitos orales deformantes, hábitos orales, dentición temporaria, dentición mixta, maloclusión, como resultado de esta primera búsqueda se obtuvieron 90 artículos.
Se revisaron los títulos, resúmenes y parte del contenido de cada uno de los documentos adquiridos, y en base a los criterios de experticia de los profesionales, se identificaron artículos considerados como sólidos, que permitieron seleccionar y desarrollar los principales aspectos de la temática. De esta manera se obtuvieron textos completos de los estudios que cumplían con los parámetros establecidos, el estudio se limitó a 17 artículos.

Los criterios de inclusión fueron los siguientes: Hábitos orales deformantes en preescolares y escolares, año de publicación del 2010 al 2017 y los criterios de exclusión: Metodología Incorrecta, revisiones narrativas, cartas al editor, artículos en revistas no indexadas y estudios que se encontraran fuera del rango etario establecido.

\section{RESULTADOS}

El propósito de esta investigación es proporcionar información actualizada sobre los hábitos deformantes orales, de los 90 artículos revisados se seleccionaron 17 como textos completos, ya que cum-

Tabla I. Prevalencia de los hábitos deformantes bucales en estudios realizados por diferentes autores.

\begin{tabular}{|c|c|c|c|}
\hline Autores & Título & País & $\begin{array}{l}\text { Prevalencia } \\
\text { de hábitos }\end{array}$ \\
\hline Blancol et al. & $\begin{array}{l}\text { Determinar los hábitos bucales } \\
\text { deformantes en estudiantes de la } \\
\text { Escuela Primaria "Raúl Gómez", } \\
\text { municipio Güines. }\end{array}$ & Cuba & $78,18 \%$ \\
\hline Murrieta-Pruneda et al., (2015) & $\begin{array}{l}\text { Prevalencia de hábitos bucales } \\
\text { parafuncionales en niños de edad } \\
\text { preescolar en Ciudad } \\
\text { Nezahualcóyotl, Estado de México. }\end{array}$ & México & $68,2 \%$ \\
\hline Chamorro et al., (2016) & $\begin{array}{l}\text { Hábitos orales frecuentes en } \\
\text { pacientes del área de } \\
\text { Odontopediatría de la Universidad } \\
\text { del Valle }\end{array}$ & Colombia & $67 \%$ \\
\hline Carillo et al., (2014) & $\begin{array}{l}\text { Hábitos bucales no fisiológicos y } \\
\text { maloclusión en niños que asisten a } \\
\text { la consulta de odontopediatría }\end{array}$ & Venezuela & $56 \%$ \\
\hline Carrillo et al., (2016) & $\begin{array}{l}\text { Relación de la succión no nutritiva } \\
\text { con la maloclusión en niños } \\
\text { ecuatorianos. Odontología. }\end{array}$ & Ecuador & $52,6 \%$ \\
\hline
\end{tabular}


plían con los parámetros establecidos de selección, solo 4 de ellos, fueron sobre estudios realizados en Ecuador.

En la Tabla I, podemos observar la prevalencia de los hábitos deformantes orales a través de datos obtenidos de trabajos realizados por diferentes autores, en países de América Latina y el Caribe. Se visualiza que el país con mayor prevalencia de hábitos orales deformantes es Cuba con un $78,18 \%$, seguido por México y Colombia con un $68,2 \%$ y $67 \%$, y Venezuela con un $56 \%$, mientras que en Ecuador la prevalencia de hábitos es menor con un $52,6 \%$, en base a nuestro criterio y experiencia profesional esto puede estar asociado a la poca información de estudios actualizados que respalden este tema.

La Tabla II, nos muestra los principales hábitos deformantes orales en diferentes países de América Latina y el Caribe, los autores de los estudios que se tomaron como referencia coincidieron que el hábito que se observa con mayor frecuencia en grupos preescolares y escolares es la Onicofagia, en países como Venezuela con un $58 \%$, en México y Colombia con un $35 \%$ y $24 \%$ respectivamente.

La disfunción lingual tuvo valores significativos, alcanzando un $38,34 \%$ en países como cuba, 19,48 $\%$ en Perú; sin dejar a un lado otros hábitos que reflejaron también incidencias significativas, como la respiración oral con un $36 \%$ y la succión digital con un $23,07 \%$ en Colombia. Estos datos porcentuales se acercan a valores publicados en estudios realizados en Ecuador y Venezuela con un $21,6 \%$ y $12 \%$, de pacientes portadores del hábito de succión digital.

Tabla II. Principales hábitos bucales deformantes.

\begin{tabular}{lcc}
\hline País & Hábitos orales Deformantes & $\%$ \\
\hline Cuba & Disfunción lingual & 38,34 \\
México & Succión digital & 23,07 \\
& Onicofagía & 35 \\
Colombia & Protracción Lingual & 6,2 \\
& Respiración bucal & 36 \\
Venezuela & Onicofagía & 24 \\
& Onicofagia & 58 \\
& Uso de biberón & 26 \\
Ecuador & Succión digital & 12 \\
Peń & Succión digital & 21,6 \\
& Deglución atípica & 19,48
\end{tabular}

Fuente: Elaboración propia a partir de los autores seleccionados.

\section{DISCUSIÓN}

La mayoría de los artículos revisados hacen referencia a la relación que existe entre los hábitos deformantes orales y las maloclusiones, siendo considerados estos uno de los principales factores etiológicos que provocan las maloclusiones.

En la Tabla I, se aprecia una alta prevalencia de los hábitos deformantes orales, lo que concuerda con lo reportado por Cepero Sánchez et al., Pruneda et al. (2014) y Reyes Romagosa et al. (2014). Los resultados de estos estudios nos expresan datos similares a lo que palpamos con frecuencia en nuestro medio y en la práctica clínica, y que a nuestro entender requieren de mayor atención y educación para la salud oral dirigida fundamentalmente a los padres, con la finalidad de fomentar hábitos de vida apropiados en los niños, otorgándole una buena alimentación, adecuado calor filial y evitando conflictos intrafamiliares o situaciones difíciles de los que lo niños sean partícipes, debido a que ciertos hábitos se instauran en el individuo al tratar de calmar una necesidad emocional; estos programas educativos se deberían impartir a niños en edad escolar y a sus maestros para interceptar los hábitos que estén presentes en esta población y evitar daños a los tejidos dentomaxilofaciales, como lo expresan Ruiz Feria et al. En Ecuador se hace evidente la prevalencia significativa de hábitos orales deformantes, aunque está fue menor que en el resto de países analizados de la literatura obtenida para esta revisión, a nuestro criterio estos valores porcentuales, pueden relacionarse con la escasa información actualizada de este tema en el país.

En la Tabla II, los resultados expuestos evidencian la prevalencia de los diferentes hábitos en países de América Latina y el Caribe, el hábito que se presenta con más frecuencia en niños es la Onicofagia, seguido de la disfunción lingual, estos criterios coinciden con los datos obtenidos por otros autores (Murrieta-Pruneda et al., 2011). Desde nuestro punto de vista profesional, la realidad en nuestro medio, nos hace diferir con los valores antes mencionado, observándose más en nuestra población hábitos como empuje lingual, respiración oral y succión digital.

Considerando otros estudios, resultados similares fueron emanados en cuanto a la prevalencia alta de hábitos parafuncionales, en la provincia de Azuay en Ecuador con un 44,92 \%, sin embargo esta investigación relaciona directamente factores como la migra- 
ción y las separaciones de las familias como detonante de los hábitos deformantes orales, y como principal factor etiológico la ansiedad, siendo muy difícil de controlar y erradicar, provocando a mediano y largo plazo afectaciones localizadas en el tejido dentario y periodontal (Chumi \& Pinos, 2014).

Desde nuestro criterio de experticia en la práctica clínica odontológica con frecuencia, el hábito de succión digital es detectado, principalmente en niños de edad preescolar, asociado rápidamente a alteraciones dentofaciales.

Pérez Acosa et al. (2013) expresan que la succión digital está presente en más de $50 \%$ de los niños pequeños, resultando tan común en la infancia que llega a ser considerado normal hasta los 3 años, y así plantea los tratamientos indicados para estos casos , relacionados a la edad del individuo.

Por otro lado cabe recalcar que de los datos analizados no se obtuvieron resultados estadísticamente significativos en cuanto al sexo y la prevalencia de hábitos orales parafuncionales (Cepero Sánchez et al.; Reyes Romagosa et al.; Murrieta Pruneda et al., 2015; Carrillo Valencia et al., 2016; Espinoza Calle et al., 2016; Pruneda et al.).

PARRA, I. S. S. \& ZAMBRANO, M. A. G. Mouth deforming habits present in preschoolers and school children: systematic review. Int. J. Odontostomat., 12(2):188-193, 2018.

ABSTRACT: Mouth deforming habits are one of the principal causes for malocclusions, among the most frequent are: Digital suction, lingual push, Onychophagia, Cheilophagia, mouth breathing, and chewing objects. The objective of the study was to execute a valuation and analysis of the available scientific evidence updated during the last seven years, regarding mouth deforming habits present in preschoolers and school children between the ages of 3 to 12 years old. A systematic review of the literature found in data basis such as: SCIELO, LILACS, ACADEMIC GOOGLE, published articles from the years 2010 and 2017 regarding mouth deforming habits present in preschoolers and school children between the ages of 3 to 12 years old. There was no restriction regarding sex or the geographical zones. A very high incidence of mouth deforming habits was found in regions such as Latin America, Central America, and the Caribbean. In countries such as Cuba the incidence was alarming. The deforming habit with mayor presence in the sample under review was Onychophagia, followed by lingual dysfunction. Results like these were also found in Venezuela, Mexico and Colombia. The results from this review showed the importance to study the mouth deforming habits, which are the main cause of malocclusion. At the same time, these habits are the third cause of many oral health problems. For the most part, the articles taken as reference for the review showed that a there was no significant statistical difference between sex and the prevalence of parafunctional habit. The importance of the implementation of new investigation projects focusing on parafunctional habits in the preschoolers and school children is highlighted. This must be accompanied by educational programs in our country Ecuador, since there were very few published articles available for this study,

KEY WORDS: mouth deforming habits, oral habits, temporary dentition, mixed dentition, malocclusion.

\section{REFERENCIAS BIBLIOGRÁFICAS}

Andrade Roman, M. E. Prevalencia de la Maloclusión Dental en Clínica de Odontopediatría de la Universidad de las Américas en Niños entre 6-13 Años. Tesis. Quito, Universidad de las Américas, 2016.

Aróstica, A. \& Carrillo, L. Prevalencia de Maloclusiones y Hábitos Orales Disfuncionales en Pre-Escolares de Establecimientos Municipales de Viña del Mar. Disertación Doctoral. Viña del Mar, Universidad Andrés Bello, 2016.

Carrillo Valencia, M. I.; Tello, G. \& Navarrete Angulo, N. E. Relación de la succión no nutritiva con la maloclusión en niños ecuatorianos. Rev. Odontol., 18(1):13-9, 2016.

Catarí, P.; Carrillo, A.; Zavarce, S. \& Pérez C., M. Hábitos bucales no fisiológicos y maloclusión en niños que asisten a la consulta de odontopediatría. Acta Odontol. Venez., 52(2), 2014. Disponible en: https://www.actaodontologica.com/ediciones/2014/2/art$8 /$

Cepero Sánchez, Z. de J.; Hidalgo-Gato Fuentes, I.; Duque de Estrada Riverón, J. \& Pérez Quiñones, J. A. Intervención educativa en escolares de 5 y 6 años con hábitos bucales deformantes. Rev. Cuba. Estomatol., 44(4), 2007. Disponible en: http://scielo.sld.cu/scielo.php?script=sci_arttext\&pid=S003475072007000400007

Chamorro, A. F.; García, C.; Mejía, E.; Viveros, E.; Soto Llanos, L.; Triana, F. E. \& Valencia, C. Hábitos orales frecuentes en pacientes del área de Odontopediatría de la Universidad del Valle. Rev. CES Odontol., 29(2), 2016. Disponible en: https:// dialnet.unirioja.es/servlet/articulo?codigo $=5759180$

Chumi, T. R. \& Pinos, N. P. Prevalencia de hábitos orales, en niños de 3 a 9 años de edad, como consecuencia de la migración de los padres al extranjero, en la comunidad Callazay, Parroquia Mariano Moreno del Cantón Gualaceo Provincia del Azuay Ecuador, año 2014. Rev. Latinoam. Ortod. Odontopediatr. [Revista en Internet] 2015. Disponible en: https://www.ortodoncia.ws/ publicaciones/2015/art-18/

Espinoza Calle, I. S.; Casas Apayco, L. \& Campos Campos, K. J. Prevalencia de hábitos orales y alteraciones dentoalveolares en niños de 6 a 12 años. Odontol. Pediatr., 15(2):127-34, 2016.

Martín Zaldivar, L.; García Peláez, S.; Expósito Martín, I.; Estrada Verdeja, V. \& Pérez Llanes, Y. Deglución anormal: algunas consideraciones sobre este hábito. A. M. C., 14(6):1-10, 2010.

Murrieta Pruneda, J. F.; Martínez Hernández, L.; Morales Vázquez, J.; Linares Vieyra, C.; \& González Guevara, M. B. Frecuencia y distribución de hábitos parafuncionales en un grupo de escolares mexicanos, 2014. Rev. Nac. Odontol., 11(21):57-64, 2015 
Murrieta-Pruneda, J. F.; Allendelagua Bello, R. I.; Pérez Silva, L. E.; Juárez-López, L. A.; Linares Vieyra, C.; Meléndez Ocampo, A. F.; Zurita Murillo, V. \& Solleiro Rebolledo, M. G. Prevalencia de hábitos bucales parafuncionales en niños de edad preescolar en Ciudad Nezahualcóyotl, Estado de México, 2009. Bol. Med. Hosp. Infant. Mex., 68(1):26-33, 2011.

Núñez, B. M. E.; González, A. O.; González, N. Y. \& Madam, O. M. Determinar los hábitos bucales deformantes en estudiantes de laEscuela Primaria "Raúl Gómez", municipio Güines. Medimay, 21(2):408-18, 2015.

Pérez Acosta, K.; Reyes Suárez, V. O.; Rodríguez, Y. L. \& Espasandín González, S. Tratamiento del hábito de succión digital mediante la técnica de relajación. Rev. Cienc. Med. La Habana, 19(3):348-57, 2013.

Reyes Romagosa, D. E.; Torres Pérez, I.; Quesada Oliva, L. M.; Milá Fernández, M. \& Labrada Estrada, H. E. Hábitos bucales deformantes en niños de 5 a 11 años. MEDISAN, 18(5):606-12, 2014.

Ruiz Feria, N.; Díaz Morell, J. E.; Rodríguez Carracedo, E. M.; Álvarez Infante, E.; Vargas Morales, Y. \& Soberats Zaldívar, M. Programa educativo sobre hábitos bucales deformantes en escolares del seminternado Juan José Fornet Piña. C. C. M.,18(4):649-63, 2014.
Dirección para correspondencia

Dra. Sandra Susana Parra Iraola

Carrera de Odontología

Universidad San Gregorio De Portoviejo

Manabí

ECUADOR

Email: sandraoverlaura@hotmail.com

Recibido : 09-01-2018

Aceptado: 13-04-2018 